\title{
Bi-allelic missense $A B C A 3$ mutations in a patient with childhood ILD who reached adulthood
}

The adenosine triphosphate (ATP)-binding cassette subfamily A member 3 (ABCA3) is a transmembrane glycoprotein that uses energy of ATP hydrolysis to transport phospholipids into the lamellar bodies of type 2 alveolar epithelial cells (AEC) and regulates lung surfactant homeostasis. More than 200 mutations have already been described in $A B C A 3$, located on chromosome $16[1,2]$. Patients present with a great heterogeneity of phenotypes, from lethal neonatal respiratory distress syndrome (RDS) to childhood and rarely adult interstitial lung disease (ILD) $[3,4] . A B C A 3$ mutations-related lung disease inheritance is autosomal recessive, as it requires two disease-causing (bi-allelic) mutations, one from each parent. Mutations may be identical or different, thereby defining the patient as being homozygous or compound heterozygous, respectively. Based on their expected functional consequences, $A B C A 3$ mutations can fall into two categories: "null/severe" (consisting mostly of nonsense, frameshift, splice site mutations and large indels) and "potentially mild" (consisting mostly of missense mutations and small in-frame indels). All subjects who are homozygous or compound heterozygous for null mutations have a very poor prognosis and die with neonatal RDS unless they undergo lung transplantation before 1 year of age. The outcome of patients homozygous for a potentially mild mutation or compound heterozygous for null/potentially mild or two different potentially mild mutations is unpredictable, ranging from the development of RDS leading to death in infancy or early childhood (1-5 years of age) to the development of childhood ILD (chILD) such as chronic pneumonitis of infancy (CPI), desquamative interstitial pneumonia (DIP), nonspecific interstitial pneumonia (NSIP) and pulmonary alveolar proteinosis (PAP), all presenting a more prolonged survival ( $>5$ years old) but rarely reaching adulthood [5-8].

A 26-year-old female never-smoker was referred by her paediatrician to our ILD clinic for deteriorating dyspnoea. She had a background of chILD diagnosed by lung biopsy at the age of 5.5 years. The patient was born without complications from non-consanguineous parents and at the age of 1.5 years developed frequent lower respiratory tract infections and, at 3 years of age, partial respiratory failure and finger clubbing. Chest computer tomography revealed a diffuse parenchymal lung disease with ground-glass opacities. Extensive work-up could not assess a diagnosis, therefore, open lung biopsy was performed at 5.5 years of age. Genetic analysis was not performed at the time due to technical and financial restrictions. Histopathology showed an unspecified ILD described initially as CPI, which was reconsidered by our expert pathologist (A. Coulomb-L'Hermine) as ILD with mild type II pneumocytes hyperplasia, diffuse septal thickening with rigid septa consistent with NSIP, intra-alveolar macrophages with some foamy macrophages, without PAP and consistent with DIP (figure 1a). At that time, the patient received systemic corticosteroids without any clinical response. After gradual tapering of steroids, hydroxychloroquine was added as a second-line option. The patient showed remarkable clinical and radiological improvement as already stated in detail in a previous report [9]. More precisely, after 3 years of treatment the patient did not desaturate at the 6-min walking test $(6 \mathrm{MWT})$, participated normally at school activities, had a chest radiograph without infiltrates and had reached the 25th percentile of body weight. Treatment with hydroxychloroquine was applied for the following 18 years during which the patient continued to develop normally. Therapy was stopped at 24 years of age due to retinopathy and the patient was referred to an adult ILD clinic.

@ERSpublications

Children with $A B C A 3$ mutations may survive beyond infancy and reach adulthood. Genetic mechanisms should always be examined in adult patients with childhood onset ILD and molecular analysis should be performed accordingly in specialised referral centres. http://bit.ly/2LzMNOE

Cite this article as: Manali ED, Legendre $\mathrm{M}$, Nathan $\mathrm{N}$, et al. Bi-allelic missense $A B C A 3$ mutations in a patient with childhood ILD who reached adulthood. ERJ Open Res 2019; 5: 00066-2019 [https:// doi.org/10.1183/23120541.00066-2019].

Copyright @ERS 2019. This article is open access and distributed under the terms of the Creative Commons Attribution Non-Commercial Licence 4.0. 

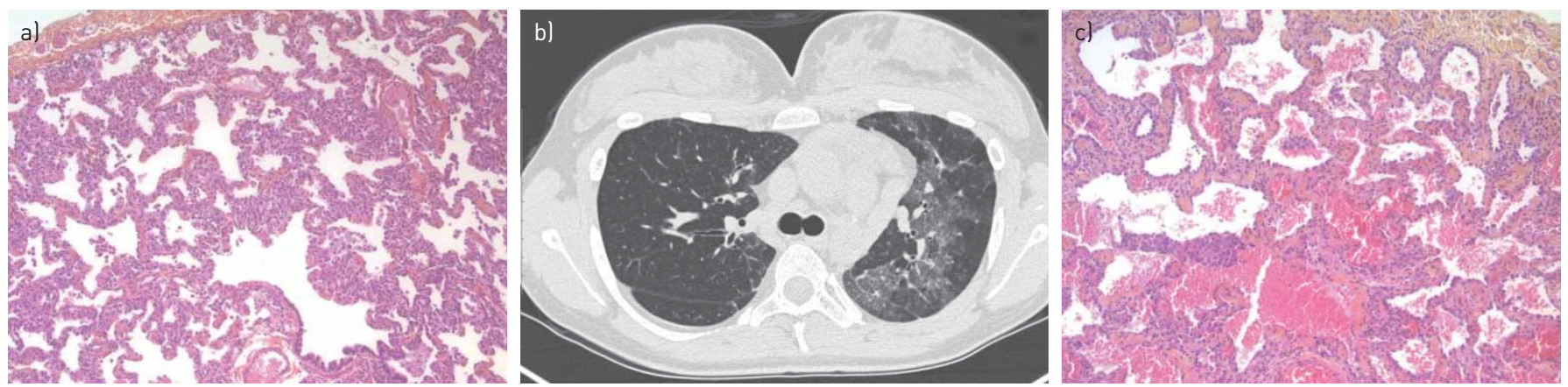

FIGURE 1 a) Haematoxylin and eosin stained view $(x 10)$ of the biopsy performed at 5.5 years of age showing mild type II pneumocytes hyperplasia, diffuse septal thickening with rigid septa but without fibrosis, and few intra-alveolar macrophages without pulmonary alveolar proteinosis. b) High-resolution computer tomography of the chest at 26 years of age revealing a diffuse parenchymal lung disease with ground-glass opacities. c) Haematoxylin and eosin stained view (x10) of the biopsy performed at 26 years of age showing a similar appearance with mild type $I I$ pneumocytes hyperplasia and diffuse septal thickening with rigid septa superimposed with fibrosis.

Upon evaluation, the patient was found to have deteriorated clinically, functionally and radiologically (figure 1b). Bronchoalveolar lavage findings were unremarkable and anti-granulocyte-macrophage colony stimulating factor antibodies were negative. To complete the diagnosis procedure, genetic testing for surfactant disorders was immediately performed. While molecular analysis was pending, the patient presented with deteriorating disease underscoring the need for imminent and appropriate therapeutic decisions. As a result, a second lung biopsy was prioritised to better classify the disease before starting empiric treatments. Histology, in comparison with the one performed 20 years earlier, found a similar appearance with mild type II pneumocytes hyperplasia and diffuse septal thickening with rigid septa superimposed with fibrosis, whereas DIP was less pronounced (figure 1c). Genetic analysis results, obtained a few months later, revealed two missense, probably pathogenic, $A B C A 3$ variations consistent with an $A B C A 3$ mutation-related ILD phenotype in a patient who reached adulthood. Family screening showed that each mutation was carried by one of her asymptomatic heterozygous parents. The variation inherited from the mother is a missense mutation located in exon 21 (c.2890G $>$ A p.(Gly964Ser)) and the one inherited from the father a missense mutation in exon 29 (c.4444C $>\mathrm{T}$ p.(Arg1482Trp)). A trial of $250 \mathrm{mg}$ azithromycin three times a week was initiated. The initial follow-up evaluation at 6 months was encouraging with stabilisation of the disease as reflected by a moderate increase in pulmonary function testing values and in the distance walked at $6 \mathrm{MWT}$. More precisely, the forced vital capacity and diffusing capacity of the lung for carbon monoxide values increased to $61 \%$ and $44 \%$ from $57 \%$ to $37 \%$ predicted, respectively, whereas the patient walked $618 \mathrm{~m}$ instead of $603 \mathrm{~m}$ at $6 \mathrm{MWT}$ desaturating to $92 \%$ instead of $89 \%$ before treatment.

A very rare case of a patient with chILD who survived to adulthood is presented here. During childhood, the patient initially failed a trial with systemic corticosteroids and was treated alternatively with hydroxychloroquine with remarkable improvement attaining stabilisation for 18 years. Withdrawal of hydroxychloroquine due to eye toxicity led to respiratory deterioration. Given the recent knowledge on chILD and adult ILD, the diagnosis was re-appraised histologically and the patient was further evaluated in order to look for a molecular cause of her disease. The diagnosis of $A B C A 3$ mutation-related ILD reaching adulthood was then established.

To our knowledge, this is the first report of an $A B C A 3$ mutation-related ILD patient with such a long-term follow-up and with two lung biopsies in a timeframe of 20 years, the first one showing the typical ABCA3 deficiency-related pattern and the second, the evolution toward interstitial fibrosis. Bi-allelic $A B C A 3$ mutation-related ILD patients surviving into adulthood are very rarely reported [10]. In one of the first studies, BulLaRD et al. [4] demonstrated that children with $A B C A 3$ mutations may survive beyond infancy and very rarely reach early adulthood. In the study of KRÖNER et al. [7], among 40 patients with two disease-causing $A B C A 3$ mutations, only two homozygotes and one compound heterozygote for potentially mild mutations, survived beyond the age of 20 years. In the study of WAMBACH et al. [6], only four potentially mild homozygous and one null/potentially mild compound heterozygous patients reaching adulthood were reported among 185 patients with bi-allelic $A B C A 3$ mutations. CAMPO et al. [11] reported a large kindred in which one adolescent was diagnosed with ILD of usual interstitial pneumonia pattern at the age of 13 years and two adult members of the same family with an unspecified ILD, mostly resembling NSIP, and all were homozygous for a potentially mild $A B C A 3$ mutation. Finally, as far as adult ILD patients are concerned, CoGHLAN et al. [12] reported an IPF adult patient homozygous for a potentially mild ABCA3 (p.Glu292Val.) mutation and Epaud et al. [13] described the first case of a 41-year-old 
patient with combined pulmonary fibrosis and emphysema, carrying null/potentially mild compound heterozygous $A B C A 3$ mutations. From the studies discussed above, it becomes evident that genotype-phenotype correlations in infants, children and very rarely adults with $A B C A 3$ mutations do exist and present with a great variation of clinical and radiological patterns ranging from fatal neonatal RDS to ILD including NSIP, DIP, PAP and much more rarely usual interstitial pneumonia and pulmonary fibrosis and emphysema $[6,7,13]$. The slowly progressive and more benign course of certain combinations of potentially mild $A B C A 3$ mutations allowing patients to reach adulthood could indeed be attributed to the lack of development of extensive deleterious fibrotic patterns as documented by the histology examination of two distant lung biopsies performed in our patient. To our knowledge, this is the first report of objective assessment by histopathology of milder disease course. Histological data from patients with ILD due to bi-allelic $A B C A 3$ mutations, who reach adulthood, are scarce and mostly regarded as non-severely fibrotic patterns, such as NSIP $[7,11]$. The two missense mutations detected in our patient are not described in the Genome Aggregation Database; they target amino acids highly conserved in vertebrates and lead to charge and steric changes in the amino acid side chain. Both mutations are, therefore, considered as probably pathogenic [14]. The combination of two such mutations with a not so severe phenotype has never been described before. However, they have already been described in the literature separately, each of them in combination with another potentially mild mutation; one in a newborn developing fatal neonatal RDS and the other one in a patient with diffuse ILD who died at 30 days of age $[6,15]$. It is worth noting that a patient previously reported with a compound heterozygous for null and p.Gly964Ser mutation (subject 68 in the study by WAMBACH et al. [6]) also had relatively milder disease, and that the adult subjects in the study by CAMPO et al. [11] had a different mutation in the same codon (p.Gly964Asp).

Besides those genotype-phenotype correlations, significant phenotypic differences could result from the contribution of other gene defects, infections and the environment. In the absence of functional studies, it is difficult to comprehend the exact mechanism of each disease-causing mutation. In general, it is known that $A B C A 3$ mutations dysregulate the normal processing and trafficking of $\mathrm{ABCA} 3$ protein and impair its ATPase activity and lipid transport function resulting in disordered lamellar body organisation and increased interleukin-8 production in AEC-2 cells $[13,16]$. Based on these observations, therapeutic interventions such as corticosteroids, hydroxychloroquine and macrolides have been widely applied to patients with $A B C A 3$ mutations, with moderate-to-very poor efficacy to date [7]. Hydroxychloroquine has been used in our patient for almost two decades and seemed to have a stabilising effect on the disease. Ophthalmological toxicity, one of the most common dosage-related side-effects, was completely reversible. Hydroxychloroquine has various immunomodulatory mechanisms of action, which include lysosomal acidification and inhibition of proteolysis, chemotaxis, phagocytosis and antigen presentation [17]. Its safety and potential efficacy in chILD are already highlighted in case reports [18] and are currently under investigation in a prospective, randomised placebo-controlled trial (clinicaltrials.gov HCQ-chILD-EU, NCT02615938). In the present report, due to corticosteroids lack of effectiveness and to hydroxychloroquine eye toxicity, azithromycin was the next therapeutic option. Azithromycin could potentially benefit $A B C A 3$ mutation-related ILD patients through its anti-inflammatory, phospholipidosis modulating and histone deacetylase-2 restoring activity [19].

To conclude, this study reports a chILD patient with bi-allelic missense $A B C A 3$ mutations who survived from childhood to adulthood. Non-progression to end-stage fibrosis was proven by a second biopsy 20 years later. Molecular diagnosis was made in adulthood and appropriate genetic counselling was provided accordingly. Genetic mechanisms should always be examined in specialised referral centres in adult patients with childhood onset ILD.

Effrosyni D. Manali ${ }^{1,10}$, Marie Legendre ${ }^{2,10}$, Nadia Nathan ${ }^{3}$, Caroline Kannengiesser $\oplus^{4}$, Aurore Coulomb-L'Hermine $\odot^{5}$, Theofanis Tsiligiannis ${ }^{6}$, Pericles Tomos ${ }^{7}$, Matthias Griese ${ }^{8}$, Raphael Borie $\odot^{9}$, Annick Clement ${ }^{3}$, Serge Amselem ${ }^{2}$, Bruno Crestani ${ }^{4,9,10}$ and Spyros A. Papiris ${ }^{1,10}$

${ }^{1}$ 2nd Pulmonary Medicine Dept, General University Hospital “Attikon”, Medical School, National and Kapodistrian University of Athens, Athens, Greece. ${ }^{2}$ Sorbonne Université, INSERM UMR_S933, and Assistance Publique Hôpitaux de Paris, Service de Génétique et d’Embryologie Médicales, Hôpital Armand Trousseau, Paris, France. ${ }^{3}$ Sorbonne Université, INSERM UMR_S933, France, Assistance Publique Hôpitaux de Paris, Service de Pneumologie Pédiatrique, Hôpital Armand Trousseau, Centre National de Référence des Maladies Respiratoires Rares RespiRare, Paris, France. ${ }^{4}$ Service de Génétique, Hôpital Bichat, Assistance Publique Hôpitaux de Paris, Université Paris Diderot, Sorbonne Paris Cité, Paris, France. ${ }^{5}$ Service d'Anatomie et de Cytologie Pathologiques, Sorbonne Université, Hôpital d'Enfants Armand Trousseau, Paris, France. ${ }^{6}$ Pediatric Pulmonology, Mitera Pediatric Hospital, Athens Greece. ${ }^{7}$ Dept of Thoracic Surgery, General University Hospital "Attikon", Medical School, National and Kapodistrian University of Athens, Athens, Greece. ${ }^{8}$ Dept of Pediatric Pneumology, Dr von Hauner Children's Hospital, 
Ludwig-Maximilians-University, German Center for Lung Research, Munich, Germany. ${ }^{9}$ Service de Pneumologie A, Hôpital Bichat, Assistance Publique Hôpitaux de Paris, Centre de Référence des Maladies Pulmonaires Rares, Université Paris Diderot, Sorbonne Paris Cité, DHU FIRE, Paris, France. ${ }^{10}$ These authors contributed equally.

Correspondence: Effrosyni D. Manali, 2nd Pulmonary Medicine Dept, General University Hospital “Attikon”, 1 Rimini Street, 12462 Haidari, Athens, Greece. E-mail: fmanali@otenet.gr

Received: May 132019 | Accepted after revision: June 032019

Conflict of interest: E.D. Manali has nothing to disclose. M. Legendre has nothing to disclose. N. Nathan has nothing to disclose. C. Kannengiesser has nothing to disclose. A. Coulomb L'Hermine has nothing to disclose. T. Tsiligiannis has nothing to disclose. P. Tomos has nothing to disclose. M. Griese reports grants from EU, during the conduct of the study; and personal fees from Boehringer and Vertex, outside the submitted work. R. Borie reports grants and personal fees from Roche and Boerhinger Ingelheim, and personal fees from Savapharma, outside the submitted work. A. Clement has nothing to disclose. S. Amselem has nothing to disclose. B. Crestani has nothing to disclose. S.A. Papiris has nothing to disclose.

Support statement: The genetic analyses are supported by grants from the Institut National de la Santé et la Recherche Médicale (INSERM), the Legs Poix from the Chancellerie des Universités (grants 2013 no. 1305, 2014 no. 1405, 2015 no. 1015, 2016 no. 2077 and 2017 no. DP2017/1860), the European Union's Seventh Framework Program (FP7-ChILD-EU 2007-2013) under grant agreement $\mathrm{n}^{\circ}$ 305653, the Bundesministerium für Bildung und Forschung (BMBF), project "HCQ4Surfdefect", under the frame of E-Rare-3, the ERA-Net for Research on Rare Diseases, as well as funding from the patient organizations Respirer c'est Grandir and Belleherbe Association. Funding information for this article has been deposited with the Crossref Funder Registry.

\section{References}

1 Dean M, Rzhetsky A, Allikmets R. The human ATP-binding cassette (ABC) transporter superfamily. Genome Res 2001; 11: 1156-1166.

2 Beers MF, Mulugeta S. The biology of the ABCA3 lipid transporter in lung health and disease. Cell Tissue Res 2017; 367: 481-493.

3 Shulenin S, Nogee LM, Annilo T, et al. ABCA3 gene mutations in newborns with fatal surfactant deficiency. N Engl J Med 2004; 350: 1296-1303.

4 Bullard JE, Wert SE, Whitsett JA, et al. ABCA3 mutations associated with pediatric interstitial lung disease. Am J Respir Crit Care Med 2005; 172: 1026-1031.

5 Flamein F, Riffault L, Muselet-Charlier C, et al. Molecular and cellular characteristics of ABCA3 mutations associated with diffuse parenchymal lung diseases in children. Hum Mol Genet 2012; 21: 765-775.

6 Wambach JA, Casey AM, Fishman MP, et al. Genotype-phenotype correlations for infants and children with ABCA3 deficiency. Am J Respir Crit Care Med 2014; 189: 1538-1543.

7 Kröner C, Wittmann T, Reu S, et al. Lung disease caused by ABCA3 mutations. Thorax 2017; 72: 213-220.

8 El Boustany P, Epaud R, Grosse C, et al. Unusual long survival despite severe lung disease of a child with biallelic loss of function mutations in ABCA-3. Respir Med Case Rep 2018; 23: 173-175.

9 Nikolaidou P, Charocopos E, Anagnostopoulos G, et al. Cellular interstitial pneumonitis in children: response to hydroxychloroquine treatment in two cases. Pediatr Asthma Allergy Immunol 2003; 16: 45-51.

10 Colin AA, Mark EJ. Case 49-1993. A 21-year-old woman with lifelong progressive interstitial lung disease. $N$ Engl J Med 1993; 329: 1797-1805.

11 Campo I, Zorzetto M, Mariani F, et al. A large kindred of pulmonary fibrosis associated with a novel ABCA3 gene variant. Respir Res 2014; 15: 43.

12 Coghlan MA, Shifren A, Huang HJ, et al. Sequencing of idiopathic pulmonary fibrosis-related genes reveals independent single gene associations. BMJ Open Respir Res 2014; 1: e000057.

13 Epaud R, Delestrain C, Louha M, et al. Combined pulmonary fibrosis and emphysema syndrome associated with ABCA3 mutations. Eur Respir J 2014; 43: 638-641.

14 Richards S, Aziz N, Bale S, et al. Standards and guidelines for the interpretation of sequence variants: a joint consensus recommendation of the American College of Medical Genetics and Genomics and the Association for Molecular Pathology. Genet Med 2015; 17: 405-424.

15 Somaschini M, Nogee LM, Sassi I, et al. Unexplained neonatal respiratory distress due to congenital surfactant deficiency. J Pediatr 2007; 150: 649-653.

16 Schindlbeck U, Wittmann T, Höppner S, et al. ABCA3 missense mutations causing surfactant dysfunction disorders have distinct cellular phenotypes. Hum Mutat 2018; 39: 841-850.

17 Braun S, Ferner M, Kronfeld K, et al. Hydroxychloroquine in children with interstitial (diffuse parenchymal) lung diseases. Pediatr Pulmonol 2015; 50: 410-419.

18 Williamson M, Wallis C. Ten-year follow-up of hydroxychloroquine treatment for ABCA3 deficiency. Pediatr Pulmonol 2014; 49: 299-301.

19 Thouvenin G, Nathan N, Epaud R, et al. Diffuse parenchymal lung disease caused by surfactant deficiency: dramatic improvement by azithromycin. BMJ Case Rep 2013; 2013: http://dx.doi.org/10.1136/bcr-2013-009988. 